\title{
Metaplastic breast cancer: a comparison between the most common histologies with poor immunohistochemistry factors
}

Salim Abraham Barquet-Muñoz', Silvia Patricia Villarreal-Colin', Luis Alonso Herrera-Montalvo², Ernesto Soto-Reyes², Carlos Pérez-Plasencia², Jaime Coronel-Martínez ${ }^{3}$, Delia Pérez-Montiel' ${ }^{4}$, Rafael Vázquez-Romo ${ }^{1}$

and David Cantú de León ${ }^{3^{*}}$

\begin{abstract}
Background: Metaplastic carcinoma of the breast $(\mathrm{MCB})$ is a rare histological type of breast cancer. This study aimed to determine whether MCB exhibits shorter overall survival (OS) and disease-free survival (DFS) compared with other histologies that are considered unfavorable.

Methods: We retrospectively analyzed 157 clinical file records of the Mexico City-based National Institute of Cancerology and compared the clinical characteristics and treatment of 24 patients with MCB, 37 patients with triple-negative invasive lobular carcinoma (TN-ILC), 48 patients with high-grade invasive ductal carcinoma (HG-IDC), and 48 patients with triple-negative invasive ductal carcinoma (TN-IDC), paired by clinical stage and age. We performed a comparative analysis and analyzed OS and DFS using a log-rank test.

Results: In patients with MCB, the 5-year DFS was 52.1\% (mean, 48.52 months; 95\%: 35.32-61.72), and the 5-year OS was $72.2 \%$ (mean, 59.77 months; $95 \% \mathrm{Cl}$ : 48.55-71.00). No differences were observed in the DFS of MCB compared with each of the other histologies (MCB vs. HG-IDC, $p=0.865$; MCB vs. TN-IDC, $p=0.966$, and MCB vs. TN-ILC, $p=0.132$ ). Moreover, no differences were observed when comparing the OS of MCB with that of each of the other histologies (MCB vs. HG-IDC, $p=0.246$; MCB vs. TN-IDC, $p=0.255$, and MCB vs. TN-ILC, $p=0.387$ ).
\end{abstract}

Conclusions: Neither OS nor DFS differ between patients with MCB and those with other histologies with unfavorable immunohistochemical factors.

Keywords: Breast, Cancer, Metaplastic, Ductal, Lobular, Triple-negative

\section{Background}

Breast cancer $(\mathrm{BC})$ is the most common malignant neoplasia in women in Mexico in terms of incidence and mortality $[1,2]$. Approximately $85 \%$ of $\mathrm{BC}$ cases involve invasive ductal carcinoma (IDC) or invasive lobular carcinoma (ILC). The remaining $15 \%$ of cases include other types of $\mathrm{BC}$ with specific differentiation, including metaplastic carcinoma of the breast (MCB) [3].

The term MCB was introduced by Huvos et al. in 1973 [4]. MCB is characterized by a heterogeneous mixture of two or three histological lineages [5,6], including adenocarcinoma and other epithelial or mesenchymal

\footnotetext{
* Correspondence: dcantude@gmail.com

${ }^{3}$ Clinical Research, Instituto Nacional de Cancerología, Mexico City, Mexico

Full list of author information is available at the end of the article
}

components $[7,8]$. Among all of the $\mathrm{BC}$ types diagnosed annually, MCB represents [9] approximately $0.25-1 \%$ [8-19].

Generally, MCB exhibits no characteristic pattern in imaging studies, is characterized by rapid growth, and requires additional immunohistochemical (IHC) studies for its adequate characterization $[5,8,11,12]$. In most cases, MCB has a triple-negative (TN) IHC profile [20-25], resulting in a less-favorable prognosis $[12,13,20,22,26]$. Optimal management of a patient with a $\mathrm{BC}$ diagnosis depends on the patient's clinical characteristics, histology, and the tumor's IHC profile [22,27]. Management can include surgery and radiotherapy (RT) for local control $[4,9,13]$. The use of systemic chemotherapy (CT) has been reported with discouraging results $[8,16,28]$. The clinicopathologic characteristics and prognosis of $\mathrm{MCB}$, compared 
with other, more common histologies, are poorly defined $[7,8,24,29,30]$. Some reports comparing the prognosis of $\mathrm{MCB}$ with those of other $\mathrm{BC}$ types suggest that $\mathrm{MCB}$ is more aggressive [7,12,22,24,29,31,32]. However, other reports have indicated that the prognosis of $\mathrm{MCB}$ is similar to that of adenocarcinoma $[8,29]$.

This study aimed to determine whether an $\mathrm{MCB}$ diagnosis confers poor prognosis with respect to overall survival (OS) and disease-free survival (DFS) compared with other common histologies that are considered unfavorable (high-grade invasive ductal carcinoma [HG-IDC], triple-negative invasive ductal carcinoma (TN-IDC), and triple-negative invasive lobular carcinoma [TN-ILC]).

\section{Methods}

Patients were selected from the database of the Mammary Tumors Department of the National Institute of Cancerology (INCan) in Mexico City between January 2005 and January 2013. We obtained the clinical file records of female patients diagnosed with MCB, IDC, or ILC using conventional histology by one of the authors. We excluded all patients with mixed histologies, those with a diagnosis of carcinoma in situ, those whose clinical file was incomplete, and those who had another histology or synchronous tumors in another organ.

From the IDC patient group, we created two subgroups, one defined as HG-IDC, to which patients with ScarffBloom-Richardson grading system 8 and 9 were assigned, and another group who had an IHC report of TN (estrogen receptors [ER] and progesterone receptors [PR] with nuclear staining of $<1 \%$, human epidermal receptor [HER2/neu] with IHC of $\leq 1$, or fluorescence in situ hybridization at a ratio of HER2:centromere enumerator probe [CEP] 17 of <1.8). We paired subjects by age and clinical stage (CS), selecting two subjects for each type of $\mathrm{MCB}$ case. From the ILC patient group, we included classic and pleomorphic variants and selected patients with an IHC report of TN as previously described.

We obtained and analyzed the following variables: age; tumor size; lymph node status; distant metastasis; CS; the presence of hormonal receptors (HRs) and HER2/neu; use of induction therapy; pathological response; surgery performed; adjuvant therapy; and recurrence, progression and survival of the patients through the last follow-up appointment or date of death. For this final variable, we located patients or spoke with a family member by telephone to determine the patient's status.

We conducted descriptive statistics for the demographic variables and report central tendency measures. We conducted univariate analysis of means using Student's $t$ test for continuous variables and the chi-square or Fisher exact test for ordinal variables.

We calculated the follow-up of each patient. DFS was defined as the period between the date at which the patient's initial multimodal treatment (surgical, radiotherapeutic, and/or systemic) terminated and the date of recurrence or the patient's last appointment. Recurrence was defined as the presence of disease $>6$ months after final treatment. Progression was defined as the presence of disease 6 months before treatment was finalized. OS was defined as the period between the date treatment ended and the date of the patient's last appointment or death.

DFS and OS were analyzed by the Kaplan-Meier survival curve method, and the curves were compared using the log-rank test. We performed multivariate analysis to ascertain which variables exerted an influence on OS and DFS using the Cox proportional hazards model. Statistical significance was defined as a $p$ value of $<0.05$. We used the statistical software program SPSS $^{\circ} 2012$ for Windows (SPSS Inc., Austin, TX, USA) for data analysis. The Director of Research of the INCan gave ethical approval for the collection and use of the data for this study.

This study was reviewed and approved by the Institutional Review Board of the National Cancer Institute of Mexico (approval number REV/20/14), and has been performed in compliance with the Helsinki Declaration. Since this study collected only archived data and materials, without using personal data from patients, we were not required by our federal legislation to obtain an informed consent from patients, as well as for phone calls, when preservation of confidentiality is assured. During the telephone call, the patient or the family member that answered was informed that a retrospective analysis was being performed and that the actual status was necessary for completing the information, also was established that all information will be kept confidential in accordance to the legislation and will be used only for this evaluation.

\section{Results}

We identified $32(0.6 \%)$ patients with a diagnosis of $\mathrm{MCB}$ among 5,440 patients diagnosed with $\mathrm{BC}$. Of these 32 patients, we excluded 8 due to incomplete clinical records or having mixed histologies, resulting in a total of 24 patients for the final analysis. Of a total of 487 patients with an ILC diagnosis, we obtained 37 (7.6\%) patients with TN-ILC. The patients with MCB were paired by age and CS at a ratio of 1:2 with patients with HG-IDC and TN-IDC, resulting in 48 patients for each group. All of the patients' general clinicopathological characteristics are described in Table 1. We analyzed 157 patients in total, with an average follow-up of 40 months (range, 2.3-97.5 months).

The 24 patients with a diagnosis of MCB had an average follow-up of 32.8 months (range, 4.1-73.4 months). The mean patient age was 49.6 years (range, 33-86 years). Three patients $(12.5 \%)$ were grade IIA, 6 (25\%) each were grade IIB, IIIA, and IIIB, 1 patient (4.2\%) was grade IIIC, 
Table 1 Clinical and pathological characteristics of patients

\begin{tabular}{|c|c|c|c|c|c|c|}
\hline & & MCB $n(\%)$ & TN-ILC $n$ (\%) & HG-IDC $n(\%)$ & TN-IDC $n(\%)$ & $p$ value \\
\hline & Number of cases & 24 & 37 & 48 & 48 & \\
\hline Age & Mean & 49.58 & 53.49 & 49.25 & 49.52 & 0.478 \\
\hline \multirow[t]{7}{*}{ CS } & I & 0 & 0 & 0 & 0 & 0.364 \\
\hline & $\| A$ & $3(12.5)$ & $4(10.8)$ & $6(12.5)$ & $6(12.5)$ & \\
\hline & $\| \mathrm{B}$ & $6(25.0)$ & $1(2.7)$ & $12(25.0)$ & $12(25.0)$ & \\
\hline & $\| I A$ & $6(25.0)$ & $13(35.1)$ & $12(25.0)$ & $12(25.0)$ & \\
\hline & $\| I B$ & $6(25.0)$ & $7(18.9)$ & $12(25.0)$ & $12(25.0)$ & \\
\hline & $\| I I C$ & $1(4.2)$ & $6(16.2)$ & $2(4.2)$ & $2(4.2)$ & \\
\hline & IV & $2(8.3)$ & $6(16.2)$ & $4(8.3$ & $4(8.3)$ & \\
\hline \multirow[t]{4}{*}{$\mathrm{T}$} & $\mathrm{T} 1$ & 0 & 0 & 0 & 0 & 0.114 \\
\hline & $\mathrm{T} 2$ & 0 & $3(8.1)$ & $1(2.1)$ & $1(2.1)$ & \\
\hline & T3 & $9(37.5)$ & $11(29.7)$ & $17(35.4)$ & 19 (39.6) & \\
\hline & T4 & $15(62.5)$ & $23(62.2)$ & $30(62.5)$ & $28(58.3)$ & \\
\hline \multirow[t]{4}{*}{ N } & NO & $7(29.2)$ & $5(13.5)$ & $9(18.8)$ & $8(16.7)$ & 0.748 \\
\hline & N1 & $8(33.3)$ & $10(27.0)$ & 19 (39.6) & $18(37.5)$ & \\
\hline & N2 & $7(29.2)$ & $16(43.2)$ & $17(35.4)$ & 19 (39.6) & \\
\hline & N3 & $2(8.3)$ & $6(16.2)$ & $3(6.3)$ & $3(6.3)$ & \\
\hline \multirow[t]{2}{*}{ M } & MO & $22(91.7)$ & $28(75.7)$ & $44(91.7)$ & $44(91.7)$ & 0.323 \\
\hline & M1 & $2(8.3)$ & $9(24.3)$ & $4(8.3)$ & $4(8.3)$ & \\
\hline \multirow[t]{2}{*}{$\mathrm{HR}$} & Estrogen & $2(8.3)$ & - & $29(60.4)$ & - & $<0.001$ \\
\hline & Progesterone & $3(12.5)$ & - & $26(54.2)$ & - & $<0.001$ \\
\hline HER2/neu & Positive & $1(4.2)$ & - & $14(29.2)$ & - & $<0.001$ \\
\hline
\end{tabular}

MCB: metaplastic carcinoma of the breast; TN-ILC: triple-negative invasive lobular carcinoma; HG-IDC: high-grade invasive ductal carcinoma; TN-IDC: triple-negative invasive ductal carcinoma; CS: clinical stage; T: tumor size; N: lymph nodes; M: distant metastasis; HR: hormone receptors; HER2/neu: human epidermal growth factor receptor 2; BC: breast cancer.

and 2 patients $(8.3 \%)$ were grade IV. All patients had tumors $>2 \mathrm{~cm}$ in size. Seven patients $(29 \%)$ had no axillary lymph node metastases, and 2 patients (8.3\%) had distant metastasis. The 48 HG-IDC patients had a mean followup of 48.3 months (range, 10.5-93.8 months), and the 48 TN-IDC patients had an average follow-up of 45.2 months (range, 5.0-97.5 months). The 37 patients diagnosed with TN-ILC had a mean follow-up of 34 months (range, 2.3-82.2 months) and a mean age of 53.49 years (range, 29-86 years). Table 2 presents the general characteristics of the treatment and clinical response.

Tables 1 and 2 show the comparative analysis of the four histologies. The presence of HR and HER2/neu was more common in HG-IDC compared with $\mathrm{MCB}$ (ER: 60.4 vs. $8.3 \% ; p<0.001$; PR: 54.2 vs. $12.5 \% ; p<0.001$; HER2/neu: 29.2 vs. $4.2 \% ; p<0.001)$. TN-IDC and HG-IDC patients were more likely to receive induction CT compared with TN-ILC and MCB patients (81.3, 79.2, 54.1, and $50 \%$, respectively; $p=0.003$ ). Regarding the type of pathological response, pathologic partial responses (pPR) were achieved by $75 \%, 65 \%, 61.5 \%$, and $16.7 \%$ of TN-ILC, HG-IDC, TN-IDC, and MCB patients, respectively $(p<0.001)$; similarly, pathologic complete responses ( $\mathrm{pCR})$ were less common in TN-ILC patients (10\%) compared with HG-IDC (30\%), TN-IDC (28.2\%), and MCB patients (25\%). Patients with MCB were more likely to progress during treatment compared with TN-ILC, TN-IDC, and HG-IDC patients $(50 \%, 10 \%, 5.1 \%$, and $5 \%$, respectively; $p<0.001)$. Among the MCB patients who experienced progression during $\mathrm{CT}$ induction, 4 received 5-fluorouraciladriamycin-cyclophosphamide (FAC) plus paclitaxel and cisplatin as a radiosensitizer plus radiotherapy; 2 received only FAC and paclitaxel. In comparison, the patients who experienced partial and complete pathological responses were treated with FAC and paclitaxel. MRM was the most frequently performed surgical procedure and was performed in $91.7 \%$ of MCB patients, $79.2 \%$ of HG-IDC patients, $77.1 \%$ of TN-IDC patients, and $64.9 \%$ of TN-ILC patients $(p=0.011)$. Patients with TN-IDC received more adjuvant CT compared with TN-ILC, MCB, and HG-IDC patients (75\%, 59.5\%, $45.8 \%$, and $39.6 \%$, respectively; $p=0.009)$. With respect to $\mathrm{RT}$, no differences were observed between MCB (32.4\%), HG-IDC (47.9\%), TN-IDC (56.3\%) and TN-ILC patients $(59.5 \%)(p=0.705)$. In the MCB group, 3 patients $(12.5 \%)$ experienced recurrence, 2 visceral and 1 to bone, whereas 
Table 2 Characteristics of treatment and response by histology

\begin{tabular}{|c|c|c|c|c|c|c|}
\hline & & MCB $n(\%)$ & TN-ILC $n(\%)$ & HG-IDC $n(\%)$ & TN-IDC $n(\%)$ & $p$ value \\
\hline & Amount received & 24 & 37 & 48 & 48 & \\
\hline Ind $C T$ & & $12(50)$ & $20(54.1)$ & $38(79.2)$ & 39 (81.3) & $<0.003$ \\
\hline \multirow[t]{4}{*}{ Response } & $\mathrm{pPR}$ & $2(16.7)$ & $15(75)$ & $26(65)$ & $24(61.5)$ & $<0.001$ \\
\hline & $\mathrm{pCR}$ & $3(25.0)$ & $2(10.0)$ & $12(30)$ & $11(28.2)$ & \\
\hline & Stable & $1(8.3)$ & $1(5)$ & 0 & $2(5.1)$ & \\
\hline & Progression & $6(50)$ & $2(10)$ & $2(5)$ & $2(5.1)$ & \\
\hline \multirow[t]{3}{*}{ Surgery } & $\mathrm{BCS}$ & 0 & 0 & $3(6.3)$ & $7(14.6)$ & 0.011 \\
\hline & MRM & $22(91.7)$ & $24(64.9)$ & $38(79.2)$ & $37(77.1)$ & \\
\hline & No & $2(8.3)$ & $13(35.1)$ & $7(14.6)$ & $4(8.3)$ & \\
\hline \multirow[t]{3}{*}{ Adjuvancy } & Received & $14(58.3)$ & $26(70.3)$ & $37(77.1)$ & $29(60.4)$ & 0.245 \\
\hline & CT & $11(45.8)$ & $22(59.5)$ & 19 (39.6) & $36(75.0)$ & 0.009 \\
\hline & RT & $14(58.3)$ & $22(59.5)$ & $23(47.9)$ & $27(56.3)$ & 0.705 \\
\hline \multirow[t]{3}{*}{ Recurrence } & No & $15(62.5)$ & $13(35.1)$ & $28(58.3)$ & $29(60.4)$ & 0.094 \\
\hline & Recurrence & $3(12.5)$ & $12(32.4)$ & $18(37.5)$ & $16(33.3)$ & \\
\hline & Progression & $6(25)$ & $12(32.4)$ & $2(4.2)$ & $3(6.3)$ & \\
\hline
\end{tabular}

MCB: metaplastic carcinoma of the breast; TN-ILC: triple-negative invasive lobular carcinoma; HG-IDC: high-grade invasive ductal carcinoma; TN-IDC: triple-negative invasive ductal carcinoma; Ind CT: induction of chemotherapy; pPR: pathologic partial response; pCR: pathologic complete response; BCS: breast-conserving surgery; MRM: modified radical mastectomy; CT: adjuvant chemotherapy; RT: radiotherapy.

6 patients (25\%) experienced progression, 5 visceral and 1 loco-regional.

In patients with $\mathrm{MCB}$, the 5-year DFS was 52.1\% (mean, 48.52 months; 95\% CI: 35.32-61.72 months), whereas the 5 -year DFS was $55.4 \%$ for HG-IDC patients (mean, 61.52 months; 95\% CI: 50.2-72.85 months), 60.1\% for TN-IDC patients (mean, 59.81 months; 95\% CI: $47.78-72.85$ months), and $29.9 \%$ for TN-ILC patients (mean, 36.13 months; 95\% CI: 25.33-46.93 months) (Table 3). When DFS was compared among all of the histologies, no significant differences were observed $(p=0.071)$ (Figure 1). The five-year OS was $72.2 \%$ for MCB patients (mean, 59.77 months; 95\% CI: 48.5571.00 months), $73.7 \%$ for HG-IDC patients (mean, 81.68 months; 95\% CI: 73.38-89.98 months), $84.8 \%$ for TN-IDC patients (mean, 85.87 months; 95\% CI: 77.10-94.64 months), and $44.3 \%$ for TN-ILC patients (mean, 56.68 months; 95\% CI: 45.63-67.74 months) (Table 3). When the OS of all of the histologies was compared, significant differences were observed $(p=0.027)$. However, when the OS of MCB was compared with each of the histologies, no significant differences for any of the comparisons were detected (MCB vs. HG-IDC, $p=0.246 ; \mathrm{MCB}$ vs. TN-IDC, $p=0.255$, and MCB vs. TN-ILC, $p=0.387$ ) (Figure 2).

We conducted a multivariate analysis with using a Cox proportional hazards model. In the comparative analysis, a significant difference $(p<0.05)$ was observed, and none of the variables independently influenced OS or DFS.

\section{Discussion}

Presently, BC is the most common neoplasm diagnosed in Mexico [2]; thus, infrequent histologies are observed more frequently than in other countries. One such histological type is MCB, and little is known about the clinicopathological characteristics and prognosis of MCB. Table 4 depicts the most representative series comparing MCB with other histologies.

In this study, $\mathrm{MCB}$ represented $0.6 \%$ of all $\mathrm{BC}$ diagnosed, a percentage similar to those reported in other studies $[5,7,10,11,22]$. In our series, the mean age at diagnosis of MCB was $>40$ years, similarly to other studies $[5,7,8,10-12,22,24,30]$. All of the MCB and other BC patients exhibited tumors $>5 \mathrm{~cm}$. Furthermore, lymph node metastasis was observed in the majority of cases, and no difference in the rate of lymph node metastasis was observed with respect to other histologies. This finding can be explained by the nature of the study and the fact that Mexican female patients tend to present at an advanced stage. Previous studies have established that MCB tends to be diagnosed with larger tumors $[7,22]$. However, Bae et al. found no difference in tumor size between MCB and TN-IDC ( $p=0.144)$ [33]. Lymph node metastasis has been shown to be less frequent in $\mathrm{MCB}$ [31,32], but not all studies have confirmed this observation $[22,33]$. In our study, $8.3 \%$ of the patient with MCB exhibited distant metastasis. However, our results with respect to $\mathrm{MCB}$ are consistent with the literature [22,31,34].

MCB was less likely to exhibit HR expression, with expression detected in $0-17 \%$ of cases $[15,16,22]$. Mourad 
Table 3 Univariate analysis of means of overall survival (OS) and disease-free survival (DFS) by histology

\begin{tabular}{lllllll}
\hline & MCB & TN-ILC & HG-IDC & TN-IDC & General & $p$ value \\
\hline OS in months (95\% Cl) & $59.77(48.55-71.00)$ & $56.68(45.63-67.74)$ & $81.68(73.38-89.98)$ & $85.87(77.10-94.64)$ & $78.66(72.64-84.68)$ & 0.027 \\
DFS in months (95\% Cl) & $48.52(35.32-61.72)$ & $36.13(25.33-46.93)$ & $61.52(50.20-72.85)$ & $59.81(47.78-71.84)$ & $55.56(48.86-62.26)$ & 0.071
\end{tabular}

95\% Cl: 95\% confidence interval; MCB: metaplastic carcinoma of the breast; TN-ILC: triple-negative invasive lobular carcinoma; HG-IDC: triple-negative invasive ductal carcinoma; TN-IDC: triple-negative invasive ductal carcinoma, OS: overall survival; DFS: disease-free survival.

et al. suggested that the absence of $\mathrm{HR}$ in $\mathrm{MCB}$ is due to the absence of an extensive glandular component [35]. Additionally, HER2/neu overexpression has been described within a range of 4-16\% [31,33]. In our study, most patients with MBC were HR-negative or HER2/neu-negative, unlike patients with HG-IDC. This description is consistent with that of other studies [12]. Park et al. described that $84 \%$ of MCB cases were TN, as were $20.1 \%$ of IDC cases $(p<0.001)$ [34].

Due to the rarity of $\mathrm{MCB}$, few studies have evaluated the response to induction therapy [12,28]. In a series of 39 patients in which 9 patients were given induction CT, 8 exhibited progression, and all died due to the disease [28]. In our study, only half of the MCB patients received CT induction. Of patients who had a response, $25 \%$ had a pCR; however, 50\% experienced progression during induction. In our institution, most patients who experience progression during induction CT have received radiotherapy with cisplatin as a radiosensitizer. Importantly, most MCB patients who exhibited progression had been treated with FAC-paclitaxel and concomitant cisplatin and radiotherapy, indicating that this histology responds poorly to this systemic treatment. This response may be associated with the intrinsic characteristics of $\mathrm{MCB}$. According to Weigelt, $\mathrm{MCB}$ can be molecularly classified as claudin-low and basal-like [36], implying a behavior and systemic therapy response similar to those of MBC and mesenchymal neoplasias [37].

The group in which more BCSs were conducted was the TN-IDC group, followed by the HG-IDC group; notably, neither MCB nor TN-ILC patients were submitted to BCS, likely because these patients were diagnosed with larger tumors and exhibited poorer responses to induction CT. Song et al. also reported more mastectomies in MCB patients relative to TN-IDC patients (92.7 vs. $80.92 \%$; $p=0.054$ ) [32]; however, Bae et al. did not observe this difference (78.7 vs. $66.1 \% ; p=0.09$ ) [33]. Of the patients who received adjuvant chemotherapy, nearly $50 \%$ received CT. This percentage can be explained by the fact that the patients who received induction CT were not included (Table 2). The percentage of patients who received RT was similar, with a slightly higher trend in MCB and TN-ILC compared with HG-IDC and TN-IDC (Table 2), possibly because BCS was less frequent

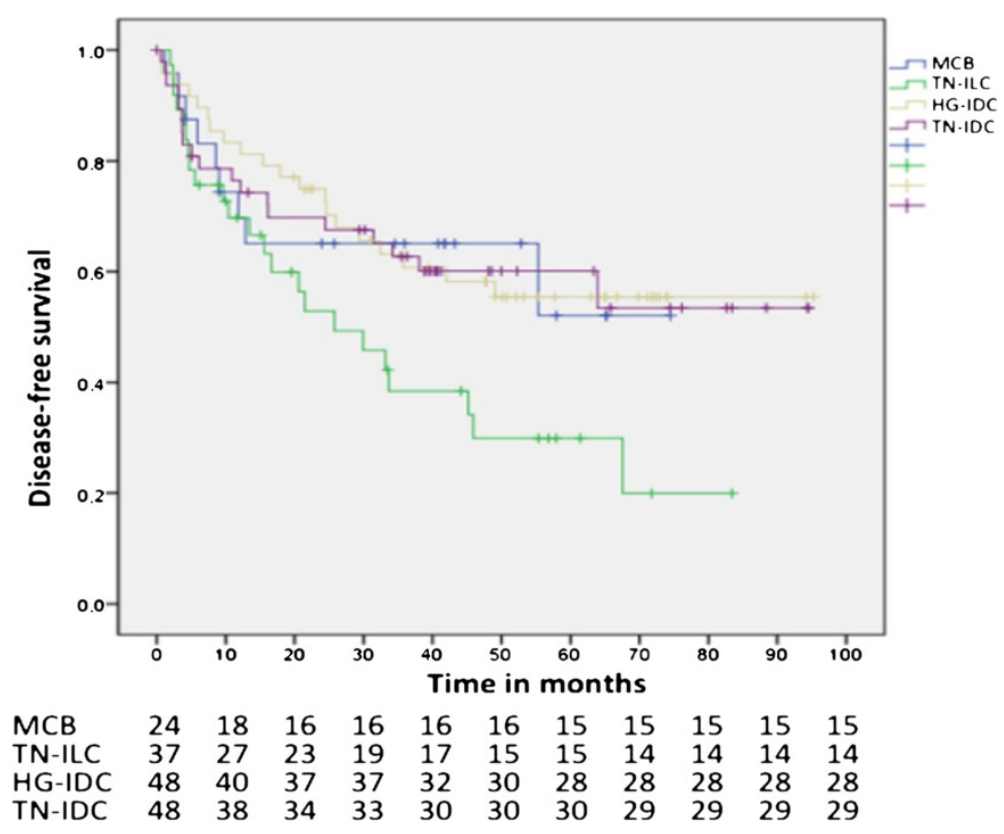

Figure 1 Disease-free survival (DFS) by individual histology. Metaplastic carcinoma of the breast (MCB) vs. high-grade invasive ductal carcinoma (HG-IDC), $p=0.865$. MCB vs. triple-negative invasive ductal carcinoma (TN-IDC), $p=0.966$. MCB vs. triple-negative invasive lobular carcinoma (TN-ILC), $p=0.132$. 


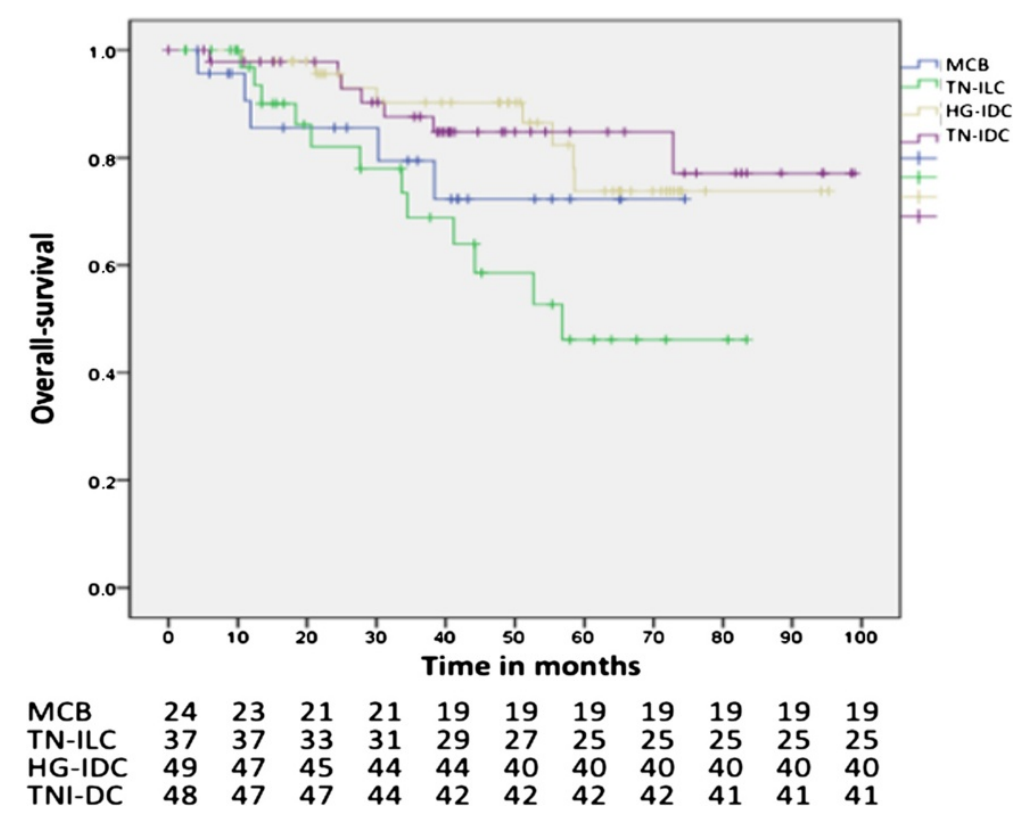

Figure 2 Overall survival (OS) by individual histology. Metaplastic carcinoma of the breast (MCB) vs. high-grade invasive ductal carcinoma (HG-IDC), $p=0.246$. MCB vs. triple-negative invasive ductal carcinoma (TN-IDC), $p=0.255$. MCB vs. triple-negative invasive lobular carcinoma (TN-ILC), $p=0.387$.

among patients with MCB and TN-ILC. There is controversy in the literature concerning whether patients with MCB tend to receive more RT than other histologies [31-33].

The mean DFS of MCB was 48.52 months, with a 5 -year DFS of $52.1 \%$. No significant differences were observed in DFS between MCB and each of the other histologies analyzed (Figure 2). The mean OS of patients with MCB was 59.77 months, with a 5 -year OS of $72.2 \%$. When comparing the OS of the groups studied, a difference was observed between the four groups $(p=0.027)$. However, when comparing the OS of MCB with each group, this difference was not detected (Figure 1). In patients with $\mathrm{MCB}$, mean OS decreased to 40 months ( 72.2 and $73 \%$, respectively); thus, patients with MCB stop dying, whereas patients with TN-ILC continue to do so (80-month OS of $72.2 \%$ vs. $44.3 \%$, respectively) (Figure 1). Notably, in our study, both MCB and TN-ILC patients exhibited worse OS compared with TN-IDC patients, making both histological types a worse prognostic factor for the OS of patients with breast cancer. Whether histology modifies OS and DFS remains controversial. In the Bae et al. study, the 3-year DFS did not differ between patients with MCB and TN-IDC $(78.1 \%$ vs. $84.9 \%$; $p<0.001)$ [3]. Lai et al. found that the 5-year DFS did not differ between MCB and ILC as much as the 5 -year OS $(p=0.289$ and 0.132 , respectively) [31]. Song et al. reported that the prognosis of MCB was worse than that of TN-IDC, with a 5-year OS of $54.5 \%$ for MCB vs. $73.3 \%$ for TN-IDC $(p<0.001)$ and a
5 -year DFS of $45.5 \%$ in MCB vs. $60.3 \%$ in TN-IDC $(p<0.001)$ [32]. Larger studies have been conducted to compare MCB and other histologies, including HR-negative patients with negative hormonal receptors, using patient information from the Surveillance, Epidemiology, and End Results from United States $[38,39]$. Nelson et al. compared 1,011 patients with MBC with 253,818 patients with IDC between 2001 and 2010; the authors also compared patients with respect to hormone receptors status and a matching analysis. The paper concludes that MBC conferred a worse OS and disease-specific survival (DSS) at 5 years compared with IDC ( $71 \%$ vs. $88 \%$, and $78 \%$ vs. $93 \%$, respectively), HR-negative cancers $(71 \%$ vs. $81 \%$ and $77 \%$ vs. $85 \%$, respectively), and the matched group ( $72 \%$ vs. $79 \%$ and $79 \%$ vs. $85 \%$, respectively) [38]. The second study was performed by Wright et al., who compared 2,338 patients with MCB with 382,667 patients with IDC; additionally, 44,813 patients with ILC were included. This study reported that patients with MCB exhibited a worse OS compared with IDC (62.1\% vs. $82.1 \%, p<0.001)$, including those who were HR-negative (63.5\% vs. $72.2 \%, p<0001)$; however, when the authors compared MCB and ILC with negative receptors, no difference was detected with respect to OS $(63.5 \%$ vs. $59.5 \%, p>0.15)$ and DSS ( $71.3 \%$ vs. $72.2 \%, p>0.65)$, similar to the TN-ILC and MCB findings in our study [39]. However, both studies compared cancers based only on the hormone receptors status, without knowing the HER2/ neu status or type of CT applied. 
Table 4 Comparison with related studies

\begin{tabular}{|c|c|c|c|c|c|c|c|c|c|c|c|c|}
\hline Author & Histology & Age (years) & & S \% & TN \% & QX \% & & & CT \% & RT \% & 5-year OS & 5-year DFS \\
\hline \multirow[t]{3}{*}{ Jung et al. (2010) } & $\mathrm{MCB} n=35$ & 47.4 & I & 17.1 || 60.0 III 8.6 IV 8.6 X 1.0 & 80.0 & MRM 48.6 BCS & $51.4 \mathrm{No}$ & 0.0 & 88.6 & 71.4 & 62.8 & 41.8 \\
\hline & IDC $n=2,839$ & 48.3 & I & 41.7 || 42.6 III 13.7 IV $2.0 \times 0.0$ & 16.7 & MRM 24.3 BCS & $74.8 \mathrm{No}$ & 1.0 & 80.6 & 82.1 & 92 & 87.3 \\
\hline & TN-IDC $n=473$ & 48.1 & I & 40.4 II 46.5 III 11.2 IV $2.1 \times 0.0$ & NA & MRM 31.7 BCS & $67.7 \mathrm{No}$ & 0.6 & 90.5 & 84.1 & 83.6 & 81.8 \\
\hline \multirow[t]{3}{*}{ Bae et al. (2011) [33] } & MCB $n=47$ & 47.0 & । & 23.4 || 70.2 III 6.4 & 93.6 & MRM $21.3 \mathrm{BCS}$ & 78.7 & & 89.4 & 80.9 & NA & 3 years 78.1 \\
\hline & IDC $n=1,346$ & 48.6 & । & 37.8 || 47.2 ||| 15.0 & 16.2 & MRM 41.0 BCS & 59.0 & & 81 & 67.4 & NA & 3 years 91.9 \\
\hline & TN-IDC $n=218$ & 47.9 & I & 32.6 || 54.6 ||| 12.8 & NA & MRM 33.9 BCS & 66.1 & & 90.9 & 73.9 & NA & 3 years 84.9 \\
\hline \multirow[t]{3}{*}{ Song et al. (2013) [32] } & $\mathrm{MCB} n=55$ & $\begin{array}{l}\leq 50=49.1 \% \\
>50=50.1 \%\end{array}$ & I & 7.3 || 54.6 III 29.1 X 9.1 & 67.3 & MRM 92.7 BCS & 7.7 & & 48 & 49.1 & 54.5 & 45.5 \\
\hline & IDC $n=767$ & $\begin{array}{l}\leq 50=52.8 \% \\
>50=47.2 \%\end{array}$ & I & 14.0 || 54.6 III 29.1 X 6.7 & 18.4 & MRM 82.5 BCS & 17.5 & & 81.7 & 23.1 & 85.1 & 71.2 \\
\hline & $\mathrm{TN}-\mathrm{IDC} n=131$ & $\begin{array}{l}\leq 50=58.0 \% \\
>50=42.0 \%\end{array}$ & 1 & 16.0 || 70.2 III 6.11 X 7.6 & NA & MRM 80.9 BCS & 19.1 & & 80.9 & 27.1 & 73.3 & 60.3 \\
\hline \multirow[t]{2}{*}{ Nelson et al. (2014) [38] } & MCB $n=1,011$ & 61 & I & 26.4 || 64.2 III 9.4 & ER/PR (-) $77.8 \%$ & MRM 53.0 BCS & 47.0 & & NA & 48.3 & $71 * 71$ & NA \\
\hline & IDC $n=253,818$ & 59 & I & 56.1 || 35.7 ||| 8.2 & ER/PR (-) 21.3\% & MRM 38.3 BCS & 61.7 & & NA & 54.3 & $88 * 81$ & NA \\
\hline \multirow[t]{3}{*}{ Wright et al. (2014) [39] } & $\mathrm{MCB} n=2,338$ & NA & । & 25.6 || 55.7 ||| 12.6 IV 6.0 & ER/PR (-) $79.0 \%$ & NA & & & NA & NA & $62.2 * 63.5$ & NA \\
\hline & IDC $n=382,667$ & NA & । & 52.2 || 30.7 ||| 12.7 IV 4.3 & ER/PR (-) $22.2 \%$ & NA & & & NA & NA & $81.2 * 72.2$ & NA \\
\hline & ILC $n=44,813$ & NA & I & 41.9 || 33.1 ||| 18.8 IV 6.3 & ER/PR (-) 3.8\% & NA & & & NA & NA & $80.2 * 59.5$ & NA \\
\hline \multirow{4}{*}{$\begin{array}{l}\text { Barquet et al. } \\
\text { (Current study) }\end{array}$} & $\mathrm{MCB} n=24$ & 49.6 & $\|$ & 37.5 III 54.2 IV 8.3 & 83.6 & MRM 91.7 BCS & $0.0 \mathrm{No}$ & 8.3 & ind CT 50.0 adj CT 45.8 & 58.3 & 72.2 & 52.1 \\
\hline & HG-IDC $n=37$ & 49.3 & $\|$ & 37.5 III 54.2 IV 8.3 & 40.0 & MRM 79.2 BCS & $6.3 \mathrm{No}$ & 14.6 & ind CT 79.2 adj CT 39.6 & 47.9 & 73.7 & 55.4 \\
\hline & TN-IDC $n=48$ & 49.5 & $\|$ & 37.5 III 54.2 IV 8.3 & NA & MRM 77.1 BCS & 14.6 No & 8.3 & ind $C T 81.3$ adj CT 75 & 56.3 & 84.8 & 60.1 \\
\hline & TN-ILC $n=48$ & 53.5 & $\|$ & 13.5 III 70.2 IV 16.2 & NA & MRM 64.9 BCS & $0.0 \mathrm{No}$ & 35.1 & ind CT 54.1 adj CT 59.5 & 59.5 & 44.3 & 29.9 \\
\hline
\end{tabular}

CS: clinical stage; TN: triple negative; QX: type of surgery; CT: chemotherapy; RT: radiotherapy; OS: overall survival; DFS: disease-free survival; MCB: metaplastic carcinoma of the breast; IDC: invasive ductal carcinoma; TN-IDC: triple-negative invasive ductal carcinoma; ILC: invasive lobular carcinoma; ER/PR(-): negative estrogen and progesterone receptors; HG-IDC: high-grade invasive ductal carcinoma; TN-IDC: triple-negative invasive ductal carcinoma; TN-ILC: triple-negative invasive lobular carcinoma: X: not known; NA: not applicable; MRM: modified radical mastectomy; BCS: breast conservative surgery; ind CT: induction chemotherapy: adj CT: adjuvant chemotherapy; ${ }^{*} \mathrm{OS}$ in ER(-)/PR(-). 
To our knowledge, this is the first study on MCB reported in the literature in Latin America and particularly in a Mexican population; furthermore, this is the first study to compare MCB with TN-ILC. Our study has several limitations, such as its retrospective design, the number of MCB cases, the focus only on IHC characteristics, and the relatively short mean follow-up. Therefore, future studies should consider the molecular characteristics of each of the tumors.

\section{Conclusions}

$\mathrm{MCB}$ is an infrequent entity and thus is rarely studied. MCB tends to exhibit less BCS, likely due to the $\mathrm{CS}$ at which it is diagnosed, its $\mathrm{TN}$ receptors and because it exhibits disease progression. However, our study demonstrated that the OS and DFS in patients with MCB do not differ from those of patients with the most common $\mathrm{BC}$ histologies with poor IHC profiles. Future studies should determine whether the molecular characteristics contribute to the prognosis of this type of $\mathrm{BC}$.

\begin{abstract}
Abbreviations
MCB: Metaplastic carcinoma of the breast; OS: Overall survival; DFS: Disease-free survival; TN-ILC: triple-negative invasive lobular carcinoma; HG-IDC: High-grade invasive ductal carcinoma; TN-IDC: Triple-negative invasive ductal carcinoma; BC: Breast cancer; IDC: Invasive ductal carcinoma; ILC: Invasive lobular carcinoma; IHC: Immunohistochemical; TN: Triple negative; RT: radiotherapy; CT: Chemotherapy; INCan: National Institute of Cancerology; ER: Estrogen receptors; PR: Progesterone receptors; HER2/ neu: Human epidermal receptor; CS: Clinical stage; HR: Hormonal receptors; pPR: Pathologic partial response; pCR: Pathologic complete response.
\end{abstract}

\section{Competing interests}

The authors declare that they have no competing interests.

\section{Authors' contributions}

$B S A, V S P$ and CD developed the original idea for the manuscript; BSA and CD wrote the first draft; HLA performed the statistical analysis; VSP, SE and PC contributed to the design of the study; PD performed the pathology review; VR collected the data; and CJ performed the first correction of the draft. All of the authors contributed to the final version of the manuscript.

\section{Authors' information}

BSA is in his last year of Gynecologic Oncology fellowship in the INCan.VSP and VR are physicians in the Department of Breast Tumors in the INCan. PD is physician in the Department of Surgical Pathology in the INCan. HLA is the chief of the Direction of Research in the INCan. PC and SE are professors in the Direction of Research in the INCan. CD is the chief of the Clinical Research Department in the INCan. CJ is professor in the Clinical Research Departament in the INCan.

\section{Acknowledgements}

The authors would like to thank César Javier Mejía Gómez, Sarish del Real Ordoñez and Milagros Pérez Quintanilla for their valuable support through the elaboration of the study.

\section{Author details}

'Department of Breast Tumors, Instituto Nacional de Cancerología, Mexico City, Mexico. ${ }^{2}$ Direction of Research, Instituto Nacional de Cancerología, Mexico City, Mexico. ${ }^{3}$ Clinical Research, Instituto Nacional de Cancerología, Mexico City, Mexico. ${ }^{4}$ Department of Surgical Pathology, Instituto Nacional de Cancerología, Mexico City, Mexico.
Received: 11 July 2014 Accepted: 10 February 2015

Published online: 20 February 2015

\section{References}

1. Ferlay J, Soerjomataram I, Ervik M, Dikshit R, Eser S, Mathers C, Rebelo M, Parkin DM, Forman D, Bray F (2012): GLOBOCAN 2012 v1. 0, Cancer incidence and mortality worldwide: IARC CancerBase No. 11. Lyon, France: International Agency for Research on Cancer. http://globocan.iarc.fr.

2. Villarreal-Garza C, Aguila C, Magallanes-Hoyos MC, Mohar A, Bargalló E, Meneses $\mathrm{A}$, et al. Breast cancer in young women in Latin America: an unmet, growing burden. Oncologist. 2013;18:1298-306.

3. Harvey JA. Unusual breast cancers: useful clues to expanding the differential diagnosis. Radiology. 2007;242:683-894.

4. Catroppo JF, Lara JF. Metastatic metaplastic carcinoma of the breast (MCB): an uncharacteristic pattern of presentation with clinicopathologic correlation. Diagn Cytopathol. 2001;25:285-91.

5. Beatty JD, Atwood M, Tickman R, Reiner M. Metaplastic breast cancer: clinical significance. Am J Surg. 2006;191:657-64.

6. Tse GM, Tan PH, Putti TC, Lui PC, Chaiwun B, Law BK. Metaplastic carcinoma of the breast: a clinicopathological review. J Clin Pathol. 2006;59:1079-83.

7. Pezzi CM, Patel-Parekh L, Cole K, Franko J, Klimberg VS, Bland K. Characteristics and treatment of metaplastic breast cancer: analysis of 892 cases from the National cancer data Base. Ann Surg Oncol. 2007;14:166-73.

8. Gibson GR, Qian D, Ku JK, Lai LL. Metaplastic breast cancer: clinical features and outcomes. Am Surg. 2005;71:725-30.

9. Tseng WH, Martínez SR. Metaplastic breast cancer: to radiate or not to radiate? Ann Surg Oncol. 2011;18:94-103.

10. Yamaguchi R, Horii R, Maeda I, Suga S, Makita M, Iwase T, et al. Clinicopathologic study of 53 metaplastic breast carcinomas: their elements and prognostic implications. Hum Pathol. 2010;41:679-85.

11. Park JM, Han BK, Moon WK, Choe YH, Ahn SH, Gong G. Metaplastic carcinoma of the breast: mammographic and sonographic findings. J Clin Ultrasound. 2000;28:179-86.

12. Luini A, Aguilar M, Gatti G, Fasani R, Botteri E, Brito JA, et al. Metaplastic carcinoma of the breast, an unusual disease with worse prognosis: the experience of the European Institute of Oncology and review of the literature. Breast Cancer Res Treat. 2007;101:349-53.

13. Dave G, Cosmatos H, Do T, Lodin K, Varshney D. Metaplastic carcinoma of the breast: a retrospective review. Int J Radiat Oncol Biol Phys. 2006;64:771-5.

14. Tavassoli FA, Devilee P. The WHO Classification of Tumors. Pathology and Genetics of Tumours of the Breast and Female Genital Organs. Lyon, France: IARC Press; 2003

15. Wargotz ES, Norris HJ. Metaplastic carcinomas of the breast. I. Matrix-producing carcinoma. Hum Pathol. 1989;20:628-35.

16. Wargotz ES, Deos PH, Norris HJ. Metaplastic carcinomas of the breast. II. Spindle cell carcinoma. Hum Pathol. 1989;20:732-40.

17. Wargotz ES, Norris HJ. Metaplastic carcinomas of the breast III. Carcinosarcoma. Cancer. 1989:64:1490-9.

18. Wargotz ES, Norris HJ. Metaplastic carcinomas of the breast IV Squamous cell carcinoma of ductal origin. Cancer. 1990;65:272-6.

19. Wargotz ES, Norris HJ. Metaplastic carcinomas of the breast: V. Metaplastic carcinoma with osteoclastic giant cells. Hum Pathol. 1990;21:1142-50.

20. Rakha EA, Ellis IO. Triple-negative/basal-like breast cancer: review. Pathology. 2009;41:40-7.

21. Gilbert JA, Goetz MP, Reynolds CA, Ingle JN, Giordano KF, Suman VJ, et al. Molecular analysis of metaplastic breast carcinoma: high EGFR copy number via aneusomy. Mol Cancer Ther. 2008;7:944-51

22. Jung SY, Kim HY, Nam BH, Min SY, Lee SJ, Park C, et al. Worse prognosis of metaplastic breast cancer patients than other patients with triple-negative breast cancer. Breast Cancer Res Treat. 2010;120:627-37.

23. Vela-Chávez TA, Ruvalcaba-Limón E, Pérez-Sánchez VM. EGFR analysis in metaplastic carcinoma of the breast [Abstract]. Eur J Cancer. 2008;6:86.

24. Al Sayed AD, El Weshi AN, Tulbah AM, Rahal MM, Ezzat AA. Metaplastic carcinoma of the breast clinical presentation, treatment results and prognostic factors. Acta Oncol. 2006;45:188-95.

25. Montagna E, Maisonneuve P, Rotmensz N, Cancello G, lorfida M, Balduzzi A, et al. Heterogeneity of triple-negative breast cancer: histologic subtyping to inform the outcome. Clin Breast Cancer. 2013;13:31-9.

26. Lee H, Jung SY, Ro JY, Kwon Y, Sohn JH, Park IH, et al. Metaplastic breast cancer: clinicopathological features and its prognosis. J Clin Pathol. 2012;65:441-6 
27. Theriault RL, Carlson RW, Allred C, Anderson BO, Burstein HJ, Edge SB, et al. Breast cancer, version 3.2013: featured updates to the NCCN guidelines. J Natl Compr Canc Netw. 2013;11:753-60.

28. Lu Y-S, Huang C-S, Cheng A-L. Poor response to systemic chemotherapy in metaplastic carcinoma of breast. EJC Suppl. 2009;7:289.

29. Chao TC, Wang CS, Chen SC, Chen MF. Metaplastic carcinomas of the breast. J Surg Oncol. 1999;71:220-5.

30. Rayson D, Adjei AA, Suman VJ, Wold LE, Ingle JN. Metaplastic breast cancer: prognosis and response to systemic therapy. Ann Oncol. 1999;10:413-9.

31. Lai HW, Tseng LM, Chang TW, Kuo YL, Hsieh CM, Chen ST, et al. The prognostic significance of metaplastic carcinoma of the breast (MCB)-a case controlled comparison study with infiltrating ductal carcinoma. Breast. 2013:22:968-73.

32. Song Y, Liu X, Zhang G, Song H, Ren Y, He X, et al. Unique clinicopathological features of metaplastic breast carcinoma compared with invasive ductal carcinoma and poor prognostic indicators. World J Surg Oncol. 2013;11:129.

33. Bae SY, Lee SK, Koo MY, Hur SM, Choi MY, Cho DH, et al. The prognoses of metaplastic breast cancer patients compared to those of triple-negative breast cancer patients. Breast Cancer Res Treat. 2011;126:471-8.

34. Park HS, Park S, Kim JH, Lee JH, Choi SY, Park BW, et al. Clinicopathologic features and outcomes of metaplastic breast carcinoma: comparison with invasive ductal carcinoma of the breast. Yonsei Med J. 2010;51:864-9.

35. Mourad WA. Book review: Rosen's breast pathology. Ann Saudi Med. 1998;18:278

36. Weigelta B, Geyerb FC, Reis-Filhob JS. Histological types of breast cancer: how special are they? Mol Oncol. 2010;4:192-208.

37. Chen IC, Lin CH, Huang CS, Lien HC, Hsu C, Kuo WH, et al. Lack of efficacy to systemic chemotherapy for treatment of metaplastic carcinoma of the breast in the modern era. Breast Cancer Res Treat. 2011;130:345-51.

38. Nelson RA, Guye ML, Luu T, Lai LL: Survival outcomes of metaplastic breast cancer patients: results from a US population-based analysis. Ann Surg Oncol 2014. [Epub ahead of print]. doi: 10.1245/s10434-014-3890-4

39. Paul Wright G, Davis AT, Koehler TJ, Melnik MK, Chung MH. Hormone receptor status does not affect prognosis in metaplastic breast cancer: a population-based analysis with comparison to infiltrating ductal and lobular carcinomas. Ann Surg Oncol. 2014;21:3497-503.

\section{Submit your next manuscript to BioMed Central and take full advantage of:}

- Convenient online submission

- Thorough peer review

- No space constraints or color figure charges

- Immediate publication on acceptance

- Inclusion in PubMed, CAS, Scopus and Google Scholar

- Research which is freely available for redistribution 